\title{
AVALIAÇÃO ESTRUTURAL DA LIGA DE NITI RICA EM NI REFUNDIDA POR PROCESSO VAR E SOLIDIFICADA EM CADINHO DE COBRE*
}

\author{
Fabiana da Silva Santos ${ }^{1}$ \\ Andersan dos Santos Paula ${ }^{2}$ \\ Patrícia Freitas Rodrigues ${ }^{3}$ \\ Francisco Manuel Braz Fernandes ${ }^{4}$ \\ Shimeni Baptista Ribeiro 5 \\ Edir Neves Teixeira ${ }^{6}$
}

\section{Resumo}

O presente estudo fez uso de uma liga de $\mathrm{NiTi}$, rica em $\mathrm{Ni}$, elaborada em um forno de fusão por indução a vácuo - VIM e posteriormente refundida em fornos de arco elétrico com eletrodo não consumível de tungstênio - VAR. Com base nos resultados de Calorimetria Diferencial de Varredura (DSC), Difração de Raios-X (DRX) e Microscopia Eletrônica de Varredura (MEV) / Espectroscopia de Energia Dispersiva de Raios-X (EDS), foi possível observar a heterogeneidade composicional/ típica de fundido por processo convencionais, as inclusões de TiC e $\mathrm{Ti}_{4} \mathrm{Ni}_{2} \mathrm{O}$ provenientes da fusão no forno $\mathrm{VIM}$, e o refinamento microestrutural e manutenção da sua composição original com uso de cadinho de cobre e câmara/atmosfera dos fornos VAR.

Palavras-chave: Liga de NiTi rica em Ni; VIM; VAR; Microestrutura.

\section{STRUCTURAL EVALUATION OF NI-RICH NITI ALLOY REMELTED BY VAR PROCESS AND SOLIDIFIED IN COPPER CRUCIBLE}

\section{Abstract}

The present study made use of a Ni-rich $\mathrm{Ni}$ alloy, developed in a vacuum induction melting furnace - VIM and subsequent remelted in vaccum arc remelting furnaces with not consumable tungsten electrode - VAR. Based on the results of Differential Scanning Calorimetry (DSC), X-ray Diffraction (XRD) and Scanning Electron Microscopy (SEM) / X-ray Dispersive Energy Spectroscopy (EDS), it was possible to observe the typical compositional heterogeneity of melted by conventional processes, $\mathrm{TiC}$ and $\mathrm{Ti}_{4} \mathrm{Ni}_{2} \mathrm{O}$ inclusions from the melting in the VIM furnace, and the microstructural refinement and maintenance of its original composition with the use of copper crucible and VAR furnaces chamber / atmosphere.

Keywords: Ni-rich NiTi alloy; VIM; VAR; Microstructure.

1 Eng. Metalurgista, Mestranda do Programa de Pós-graduação em Ciência dos Materiais do Programa de Pós-graduação em Ciencia dos Materiais (PGCM), Seção de Engenharia Mecânica e de Materiais (SE-4), Instituto Militar de Engenharia, Rio de Janeiro, RJ, Brasil.

2 Eng. Metalurgista, M.Sc., D.Sc., Professora Adjunta (PGCM / SE-4), IME, Rio de Janeiro, RJ, Brasil.

3 Bacharel em Química Industrial, M.Sc., Doutoranda em Ciência dos Materiais, Centro de Investigação de Materiais (CENIMAT/i3N), Departamento de Ciência dos Materiais (DCM), Faculdade de Ciência e Tecnologia (FCT), Universidade Nova de Lisboa (UNL), Caparica, Portugal.

4 Engo Mecânico, M.Sc., D.Sc., Professor Associado, CENIMAT/i3N / DCM / FCT / UNL, Caparica, Portugal.

5 Eng ${ }^{\underline{a}}$ Metalurgista, M.Sc., D.Sc., Professora, UniFOA, Volta Redonda, RJ, Brasil.

6 Engo Metalurgista, M.Sc., Professor, UniFOA, Volta Redonda, RJ, Brasil. 


\section{INTRODUÇÃO}

A produção de ligas de NiTi é feita principalmente pela fusão a vácuo, fusão por feixe de elétrons, fusão à arco [1,2], fusão a vácuo de alta frequência em um cadinho de grafite [3,4], fusão por plasma, etc. Quando as ligas de NiTi são fundidas, podem haver efeitos desfavoráveis, especialmente de gases, tais como nitrogênio ou oxigênio. Outros problemas consistem nas condições adequadas para cristalização e minimização da micro e macro segregação ligadas a isso. A liga pode também ser significativamente influenciada pelas impurezas incorporadas durante os processos de elaboração, conformação, tratamento térmico, etc. Tendo como destaque os gases $\left(\mathrm{O}_{2}, \mathrm{~N}_{2}, \mathrm{H}_{2}\right)$ e carbono. Por isso, a contaminação do material por inclusões não metálicas tem de ser evitada [5], como é o caso da formação de carboneto de titânio ( $\mathrm{TiC}$ ) e óxido complexo de titânio e níquel $\left(\mathrm{T}_{4} \mathrm{Ni}_{2} \mathrm{O}\right)$ na elaboração por fusão de ligas do sistema $\mathrm{Ni}$-Ti. No caso das ligas a base do intermetálico $\mathrm{NiTi}$, a concentração desses elementos metálicos individuais muda no intermetálico, em função do percentual de $\mathrm{C}$ e $\mathrm{O}$ presente.

1.1 VIM - Vacuum Induction Melting (Fusão por Indução a Vácuo)

O VIM é um dos processos utilizados na produção das ligas de NiTi. A tecnologia de fusão por indução a vácuo em cadinhos de grafite representa o principal método de preparação existente, mas tem uma série de desvantagens se algumas atenções não forem tomadas. A homogeneidade química dentro desta tecnologia pode ser obtida através do controle apropriado da energia (mistura da liga líquida). Quando se utiliza esta tecnologia, a qualidade da liga preparada dependerá fortemente do material do cadinho. Normalmente, o cadinho de grafite é o recomendado - onde o teor de oxigênio pode ser ausente; no entanto, a absorção de carbono deve ser considerada (há uma influência significativa do carbono sobre as características microestruturais). Durante a preparação do material num cadinho de grafite [6], verificou-se também que, no caso da utilização de pastilhas de $\mathrm{Ni}$ e barras / discos de $\mathrm{Ti}$, era importante a disposição apropriada do material no cadinho. Mesmo a superfície interna do cadinho fosse coberta com discos de $\mathrm{Ti}$, o teor de carbono na liga produzida era menor em comparação com o arranjo aleatório de carga. Este fenômeno é causado pela formação de uma camada de TiC, que atua como uma barreira de difusão. Verificou-se também que o teor de carbono depende fortemente da temperatura e do tempo de permanência da massa fundida no cadinho. Por esta razão, uma investigação mais intensiva desses efeitos foi realizada [7]. Foi estabelecido que com o aumento do tempo de permanência da fusão no cadinho o fundido fica enriquecido em carbono.

Quando o NiTi fundido entra em contato com a grafite do cadinho, ocorre a interdifusão ocasionando um crescimento da camada de TiC crescendo a quantidade de carbono na liga fundida. Este processo inclui a difusão de carbono através de uma fina camada de TiC que cresce na fronteira entre TiC / NiTi fundido.

1.1.1 VAR - Vacuum Arc Remelting (Forno a Arco de Refusão a Vácuo)

A tecnologia VAR é amplamente utilizada para aumentar a pureza metalúrgica de ligas preparadas utilizando procedimentos padrão, por exemplo, em fornos de indução a vácuo. Este procedimento é também conhecido como "eletrodos consumíveis".

A corrente contínua é utilizada para a formação do arco entre o eletrodo (material fundido) e um cadinho de cobre / molde resfriado à água. Como consequência, a ponta do eletrodo é fundida e é formado um novo lingote no interior do cadinho. Desta maneira, a fusão durante a fusão do arco a vácuo não está em contato com o 
cadinho de grafite (tal como no caso da técnica VIM com um cadinho / molde de grafite), podendo assim obter um produto "mais puro" utilizando este método [8,9].

Muitas vezes, o processo VIM + VAR é aplicado para a preparação de ligas NiTi. A tecnologia VAR também é preferida para a preparação de material experimental para elaboração das ligas de $\mathrm{NiTi}$.

Foram também desenvolvidos fornos para processo VAR em forno com cadinho de cobre refrigerado, com uso de eletrodo de tungstênio (não consumível) e utilização da carga metálica (liga previamente elaborada ou carga metálica individualizada de $\mathrm{Ni}$ e $\mathrm{Ti}$ de alta pureza), onde pela abertura de arco elétrico em função de uma atmosfera de argônio permite a fusão e elaboração da liga. A prática de múltiplas refusões permite a homogeneização do fundido, no entanto normalmente limita o volume de carga metálica a ser trabalhada. Este tipo de equipamento foi concebido na base do princípio do processo de soldagem com gás inerte e eletrodo de tungstênio não consumível (TIG). Neste trabalho foram utilizados 2 fornos seguindo esta tecnologia de eletrodo não consumível.

\section{MATERIAL E MÉTODOS}

O material a ser analisado foi uma liga de NiTi rica em Ni produzida no ITA, pelo Grupo do Pesquisador Professor Jorge Otubo, através do processo de fusão em forno de indução a vácuo (VIM) na forma de um lingote de 90 × 300 mm - diâmetro x altura, pesando aproximadamente $20 \mathrm{~kg}$ [10]. Uma fatia do lingote denominado de VIM51 foi destinada a este estudo. Segundo informações cedidas pelo professor Otubo a composição química nominal da liga é 55\%Ti, 0,05\%C, 0,084\%O em peso (p/p).

Uma fatia do lingote proveniente do processo (VIM) foi subdivida em amostras menores, com aproximadamente $90 \mathrm{~g}$, por meio de corte por eletroerosão (Empresa Elevolt em Volta Redonda / RJ), para serem refundidas no forno de fusão a arco VAR (com eletrodo não consumível de tungstênio, atmosfera de argônio e cadinho de cobre refrigerado com água) do ITA. Amostras do lingote refundido no forno de ITA, de aproximadamente $20 \mathrm{~g}$, foram novamente refundidas em outro forno VAR (com eletrodo não consumível de tungstênio, atmosfera de argônio e cadinho de cobre refrigerado com água, instalado nos laboratórios da Escola de Engenharia da Universidade Politécnica de Timisoara (UPT) na Romênia) e dotado de sistema de fundição para sucção a vácuo com molde de cobre (refrigerado com água).

Com base nos resultados de composição obtidos por Ribeiro (2015) [11], a liga foi definida como rica em $\mathrm{Ni}$ em função dos teores de $\mathrm{Ni}$ e Ti livres para formação do intermetálico NiTi. Ribeiro (2015), [11], procedeu a sua análise química com uso de um espectrômetro de comprimento de onda dispersiva de Raio-X (WDS) acoplado a um MEV de filamento de emissão de campo (FEG), detectando em média:

- 55,54\%p.Ni (49,50\%at.Ni), 43,69\%p.Ti (47,73\%at.Ti), 0,52\%p.O (1,70\%at.O) e 0,25\%p.C (1,07\%at.C) para condição bruta de fusão (VIM);

- 56,02\%p.Ni (50,10\%at.Ni), 43,31\%p.Ti (47,48\%at.Ti), 0,51\%p.O (1,68\%at.O) e 0,17\%p.C (0,74\%at.C) para condição refundida (VAR ITA).

Ribeiro (2015), procedeu a cálculos, em função da presença de carbono e oxigênio na forma de carbeto e óxido ( $\mathrm{TiC}$ e $\mathrm{Ti}_{4} \mathrm{Ni}_{2} \mathrm{O}$ - identificados por $\mathrm{DRX}$ ), do teor de $\mathrm{Ni}$ e Ti livre para formação do intermetálico, resultou em média de:

- 51,27\%at.Ni e 48,72\%at.Ti para condição bruta de fusão (VIM);

- 51,54\%at.Ni e 48,46\%at. Ti para condição refundida (VAR ITA).

Indicando dessa forma que o processo de refusão (VAR ITA) não foi prejudicial à liga do ponto de vista de incorporar mais oxigênio a mesma. 
$\mathrm{Na}$ tabela 1 são apresentadas as amostras obtidas pelas etapas de processamento VIM e VAR, selecionadas para este estudo.

Tabela 1. Amostras selecionadas para a análise.

\begin{tabular}{c|c|c}
\hline Amostras & Processamento & Massa do Lingote (g) \\
\hline AM1 & Bruta de fusão, forno VIM & $20.000 \mathrm{~g}$ \\
\hline AM3 & Refundida, forno VAR ITA & $90 \mathrm{~g}$ \\
\hline AM6 & Refundida, forno VAR UPT & $20 \mathrm{~g}$ \\
\hline
\end{tabular}

A caracterização para avaliar a evolução estrutural foi realizada através das técnicas: Calorimetria Diferencial de Varredura (DSC), microscopia eletrônica de varredura (MEV) com microanálise elementar via energia dispersiva de Raio-X (EDS), difração de raios-x (DRX).

Para a realização das análises de calorimetria diferencial de varredura (DSC) foi utilizado o equipamento DSC 204 F1 Phoenix - Calorimetria Exploratória Diferencial e Proteus Phoenix, instalado em um dos laboratórios do Centro de Investigação de Materiais (CENIMAT/i3N) da Faculdade de Ciências e Tecnologia (FCT) da Universidade Nova de Lisboa (UNL), Caparica - Portugal. As amostras foram preparadas a partir do corte de precisão com disco diamantado e posteriormente decapadas (Solução: $45 \mathrm{~mL} \mathrm{H} \mathrm{H}_{2}, 45 \mathrm{~mL} \mathrm{HNO}_{3}$, e $10 \mathrm{~mL} \mathrm{HF}$ ) para remover a camada de óxido e deformada gerada no corte. Foram realizados dois ciclos de aquecimento/resfriamento no intervalo de temperatura de -150 a $+150^{\circ} \mathrm{C}$, com taxa de aquecimento e resfriamento de $10^{\circ} \mathrm{C} / \mathrm{min}$, em atmosfera inerte de nitrogênio gasoso.

As amostras para as análises de MEV/EDS e Ultramicrodureza preparadas a parte do corte de precisão da seção transversal, associada ao diâmetro das amostras, sendo inicialmente lixadas (até 2500 mesh) e posteriormente submetidas ao polimento eletrolítico com solução $20 \%$ de ácido sulfúrico e $80 \%$ de metanol a temperatura ambiente, a tensão adotada, definida objetivando densidade de corrente de $0,8 \mathrm{~A} / \mathrm{cm}^{2}$ e o tempo em função da condição microestrutural das amostras (entre 10 a $26 \mathrm{~s})$.

Foi utilizado um Microscópio Eletrônico de Varredura - MEV FEI Quanta 250 FEG com filamento de emissão de campo (FEG) e espectrômetro de energia dispersiva de Raios-X (EDS) da Bruker, instalado no laboratório de Microscopia Eletrônica do Instituto Militar de Engenharia (IME). As análises foram conduzidas com uso dos detectores de elétrons secundários (SE) e de elétrons Retroespalhados (BSE), com uso de tensão de $20 \mathrm{kV}$, spot size de 4,5, distância de trabalho de aproximadamente $10 \mathrm{~mm}$, e abertura 5 .

A superfície de análise das amostras de DRX (mínimo de $1 \mathrm{~cm}^{2}$ de área transversal) foi preparada via sequência de lixamento adotada para as amostras de MEV e posteriormente serão submetidas a mesma solução de polimento químico utilizada para as amostras de DSC, com imersão por cerca de 30 minutos. No que diz respeito as análises de DRX:

- para amostra bruta de fusão, processo de fusão VIM - AM1, que a análise foi conduzida em um difratômetro da Rigaku (CENIMAT/i3N/FCT/UNL), com tubo de cobre $\left(\mathrm{Cu} \mathrm{K} \mathrm{K}_{\alpha}=1,5473 \hat{A}\right)$, no intervalo de 2theta de 15 a $85^{\circ}$, passe de 0,08 e tempo de aquisição de 2 segundo por ponto. 
- para as amostras refundidas (VAR ITA e VAR UPT), as análises foram conduzidas em um difratômetro de Raios-X, modelo X'PERT PRO MPD da PANalytical, com o software Data Colector - Absolute Scan, com o detector X'Celerator. Utilizou-se a configuração foco linha para a obtenção de difratogramas com boas intensidades. Os parâmetros de medida foram ângulos iniciais e finais de $30^{\circ}$ e $95^{\circ}$, respectivamente, para o ângulo de $2 \theta$, com acoplamento $\theta / 2 \theta$, passo de $0,03^{\circ}$ e tempo por passo de $300 \mathrm{~s}$.

\section{RESULTADOS E DISCUSSÃO}

A seguir, são apresentados os resultados e sua respectiva discussão comparando as condições refundidas via os fornos VAR do ITA - Brasil (AM3) e da UPT Romênia (AM6) em relação ao lingote de partida oriundo da elaboração da liga via forno VIM do ITA (AM1 - lingote inicial).

$\mathrm{Na}$ Figura 1 são mostradas as curvas de DSC das amostras na condição bruta de fusão (AM1), proveniente de um lingote de $20 \mathrm{~kg}$ em que foi fundido via processo VIM em cadinho de grafite, condição refundida para o forjamento rotativo (AM3) e condição refundida para a fundição por sucção a vácuo (AM6), as quais são provenientes de um pedaço de aproximadamente $90 \mathrm{~g} \mathrm{e} 20 \mathrm{~g}$ da amostra bruta de fusão (AM1), respectivamente, e que passaram pelo processo de refusão a arco elétrico (VAR), com eletrodo não consumível em um cadinho de cobre com atmosfera controlada de gás argônio e resfriado com água. Devido à diferença do tamanho dos lingotes, $(20 \mathrm{~kg}, 90 \mathrm{~g}$ e $20 \mathrm{~g})$ pode-se considerar que a taxa de resfriamento a que foram submetidos incrementou com o decréscimo da massa.

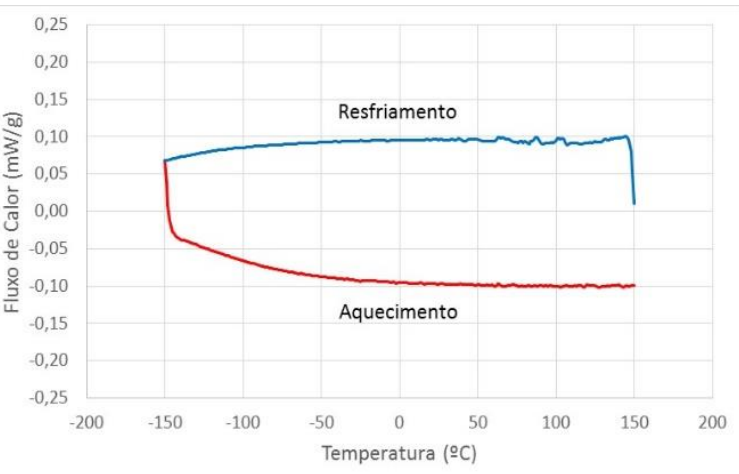

(a)

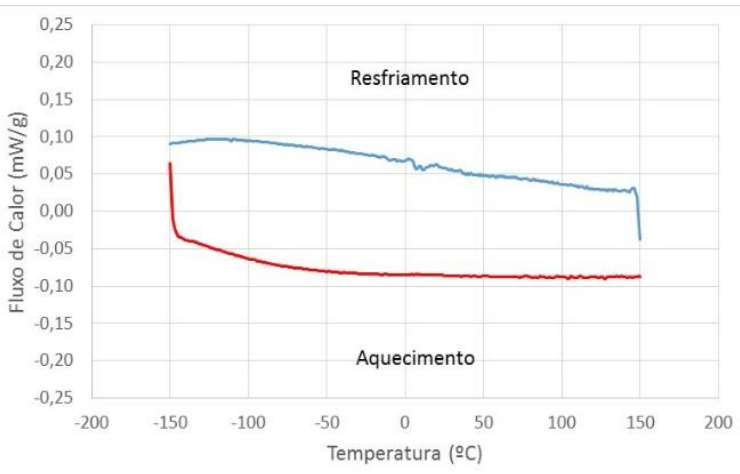

(b)

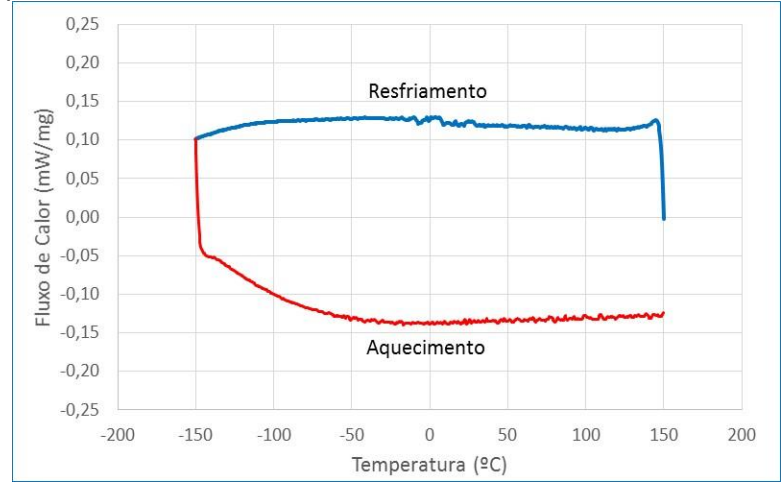

(c)

Figura 1. Curvas de DSC das amostras: (a) bruta de fusão (AM1); (b) refundida VAR ITA (AM3); e (c) refundida VAR UPT (AM6). 
É possível observar nas curvas de DSC, das três amostras da liga de NiTi em estudo, que não há presença de picos de transformação martensítica e reversa. As amostras são resultantes do processo de solidificação da liga e posterior resfriamento até a temperatura ambiente. Segundo a literatura [12], comportamento esperado pela possível heterogeneidade composicional resultante de taxas de resfriamento lentas a moderadas que resultam numa significativa heterogeneidade composicional associada à formação de distintas formas estequiométricas relacionadas aos intermetálicos de $\mathrm{Ni}$ e $\mathrm{Ti}$, para além daquele de natureza equiatômica (NiTi) que é responsável pelas transformações martensítica e reversa nestas ligas. Com isto, somente adoção de taxas de resfriamento mais aceleradas desde a solidificação (que permitam que ocorra a cristalização) ou um processamento termomecânico posterior associado ou não a um tratamento prévio para homogeneização microestrutural seriam capazes de resultar em condições estruturais adequadas a exibir as transformações em voga no produto somente fundido $[13,14]$.

A seguir, na Figura 2 são mostrados os difratogramas de DRX das amostras bruta de fusão (AM1), via processo VIM, e as refundidas via processo VAR (AM3 - ITA) e (AM6 - UPT), respectivamente.

É possível observar que para as três amostras se fez presente a fase B2 (austenita) a temperatura ambiente. Para a amostra bruta de fusão (AM1), se fez presente picos de óxidos $\left(\mathrm{Ti}_{4} \mathrm{Ni}_{2} \mathrm{O}\right)$ por ser uma amostra proveniente de fusão no forno $\mathrm{VIM}$, onde provavelmente o nível de vácuo não era tão alto para minimizar a incorporação de oxigênio ou a matéria prima utilizada para fusão continha óxido pré-existentes em nível significativo, causando a incorporação de oxigênio durante a elaboração da liga no estado líquido [5].

$\mathrm{Na}$ amostra refundida (AM3), por ser proveniente de um pedaço de $90 \mathrm{~g}$ aproximadamente da bruta de fusão (AM1) e proveniente do processo VIM, apresentou picos de inclusão do tipo carboneto de $\mathrm{Ti}$ (TiC) e precipitados de $\mathrm{Ni}_{4} \mathrm{Ti}_{3}$. Com base na literatura [7,15], quando ocorre a fusão da liga de NiTi em forno VIM, a mesma entra em contato com o cadinho de grafite ocasionando a interdifusão e promovendo a formação de $\mathrm{TiC}$ devido a afinidade que o carbono tem de se combinar com titânio. Na amostra refundida (AM6) para proveniente de aproximadamente $20 \mathrm{~g}$ da bruta de fusão (AM1) se fez presente o precipitado de $\mathrm{Ni}_{4} \mathrm{Ti}_{3}$. No entanto, qualquer óxido e até mesmo carbeto presente na matéria prima (lingote VIM) a ser refundida por um dos fornos VAR (ITA ou UPT) resultaram em lingotes refundidos com as mesmas contaminações, podendo apenas haver uma alteração na distribuição na macro/microestrutura devido a taxa de resfriamento a que cada um dos lingotes foi submetido em função da sua massa e taxa de extração de calor dos materiais do cadinho (grafite e cobre) e respectivo sistema de refrigeração, caso estes últimos se façam presentes. A justificativa para os picos distintos de segunda fase (precipitados ou inclusões) presentes em cada um dos lingotes analisados, pode estar associada a macroestrutura formada em cada um com aspecto texturais distintos para cada produto fundido nestas etapas. Baseada na literatura [16,17], a análise de DRX analisa um volume restrito de material, por ser um feixe com baixa penetração e dependendo de sua radiação ( $\mathrm{Cu} \mathrm{Ka}$, Co Ka, $\mathrm{Cr} \mathrm{Ka}$ e $\mathrm{Mo} \mathrm{Ka}$ ) a penetração é inferior a $10 \mu \mathrm{m}$. Fatores estes que poderiam vir a justificar as diferenças entre os difratogramas das 3 amostras (AM1, AM3 e AM6). 


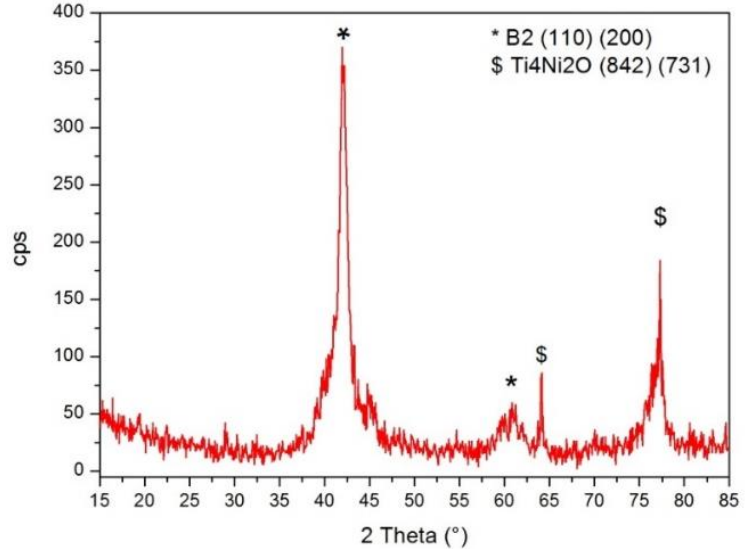

(a)

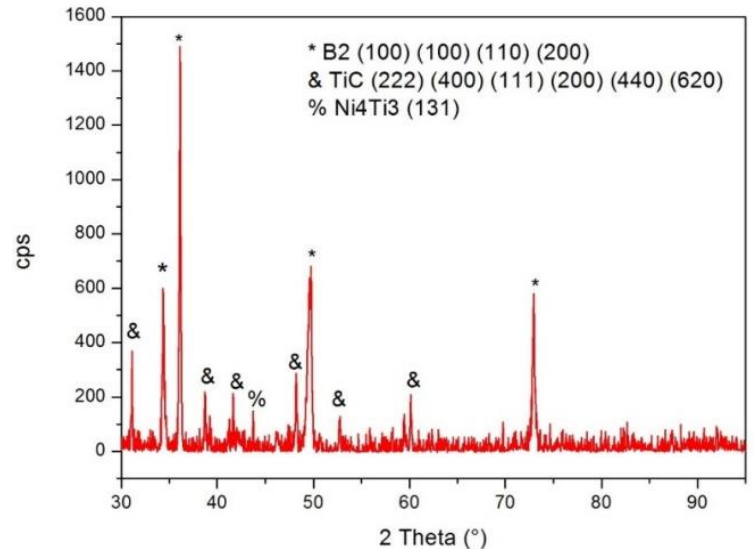

(b)

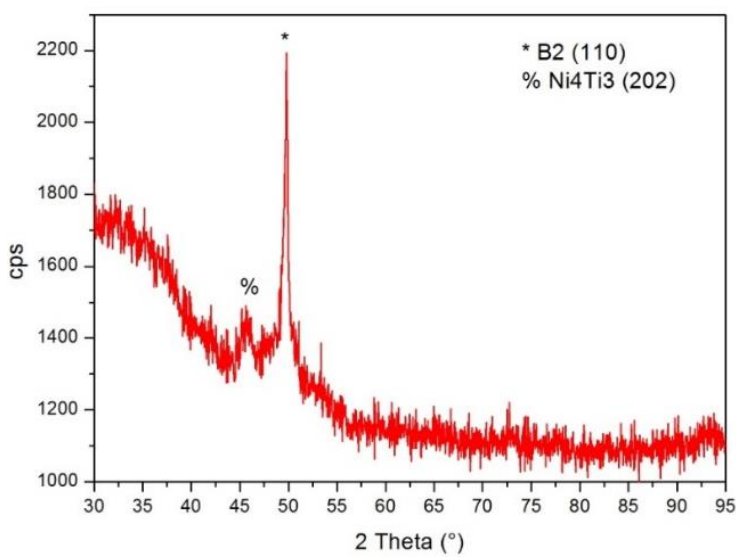

(c)

Figura 2. Difratograma de DRX das amostras: (a) bruta de fusão (AM1), Radiação Cu K $\alpha$; (b)

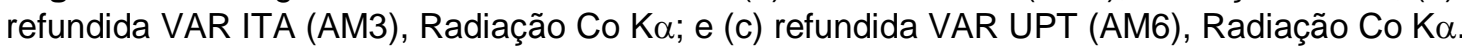

Na Figura 3 foram observados os aspectos microestruturais quanto à morfologia / distribuição de inclusões junto a matriz metálica, após polimento eletrolítico com uso do MEV (detectores de elétrons secundários (SE) e retroespalhados (BSE)), nas amostras bruta de fusão (AM1), refundida forno VAR ITA (AM3) e refundida forno VAR UPT (AM6). Foi possível observar num aspecto geral, a presença de uma matriz metálica de NiTi representada pela região mais escura e presença de inclusões representada por pontos e/ou regiões mais clara. De acordo com o trabalho de J. FRENZEL et al., [18] a solidificação em ambos os processos (VIM e VAR) começa a partir do fundo do lingote, onde a massa fundida está em contato com o cadinho de grafite e/ou com cobre refrigerado com água. Logo, a formação de óxidos pelo processo VIM está relacionada a captação de oxigênio durante a etapa de fusão, uma vez que a atmosfera não é tão bem controlada como nos fornos VAR. Assim como as diferenças significativas de $\mathrm{Ni}$ e $\mathrm{Ti}$, o baixo coeficiente de difusão do $\mathrm{Ti}$ e a afinidade que o carbono tem de se combinar, são as razões para a microssegração e formação de carbonetos (TiC). 


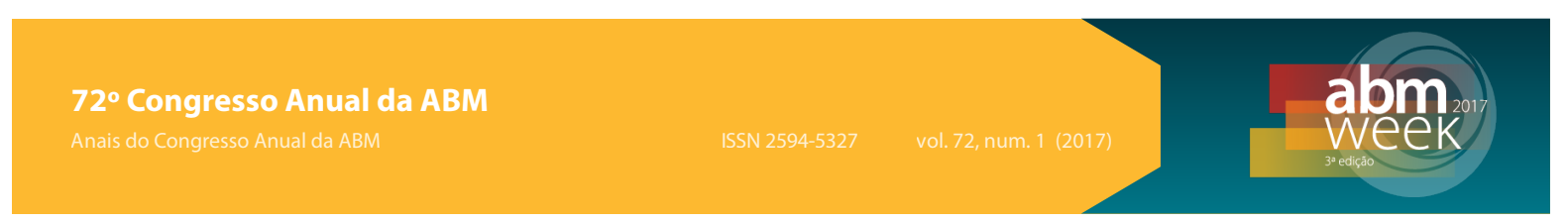

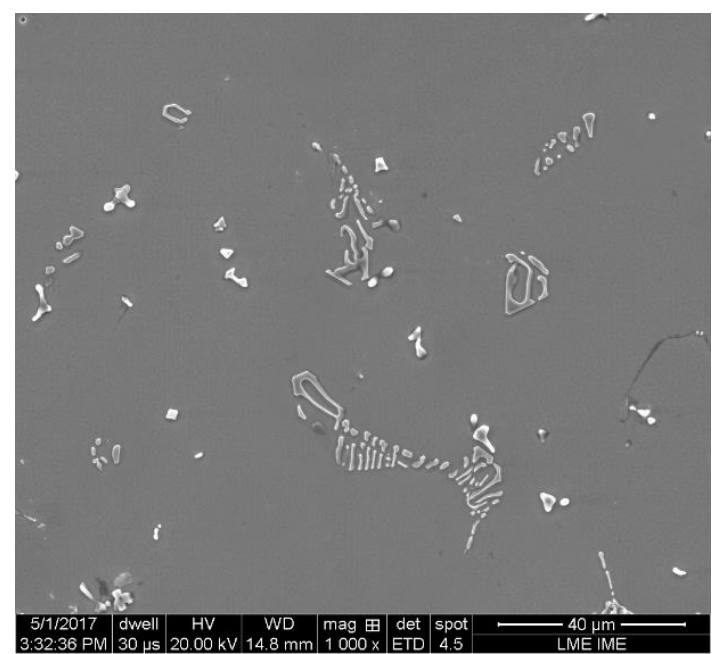

(a)

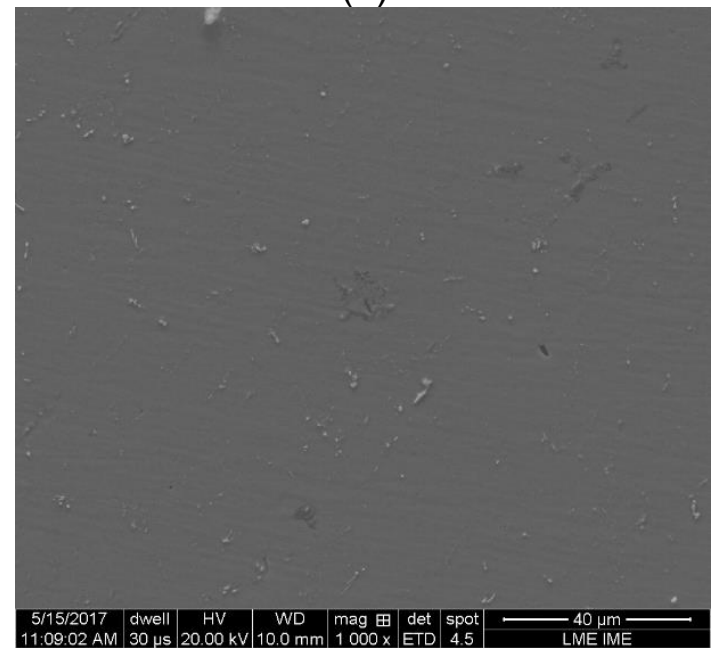

(c)

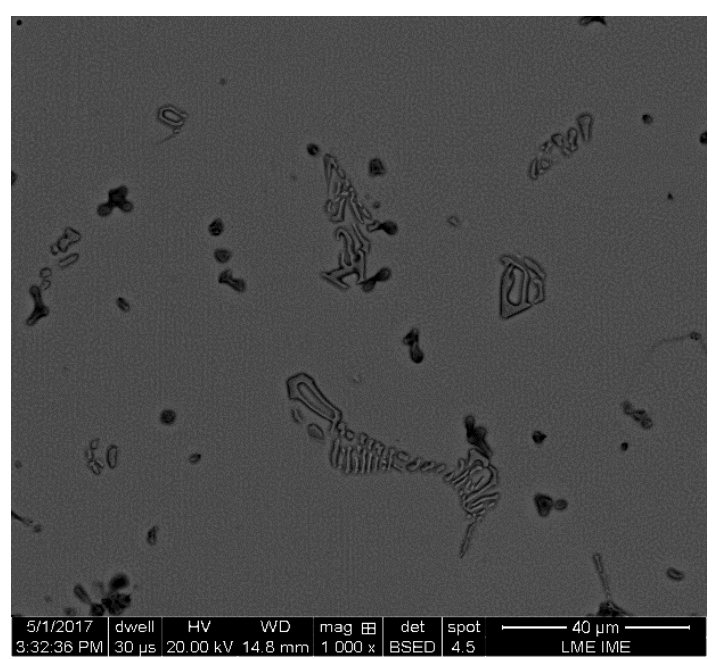

(b)

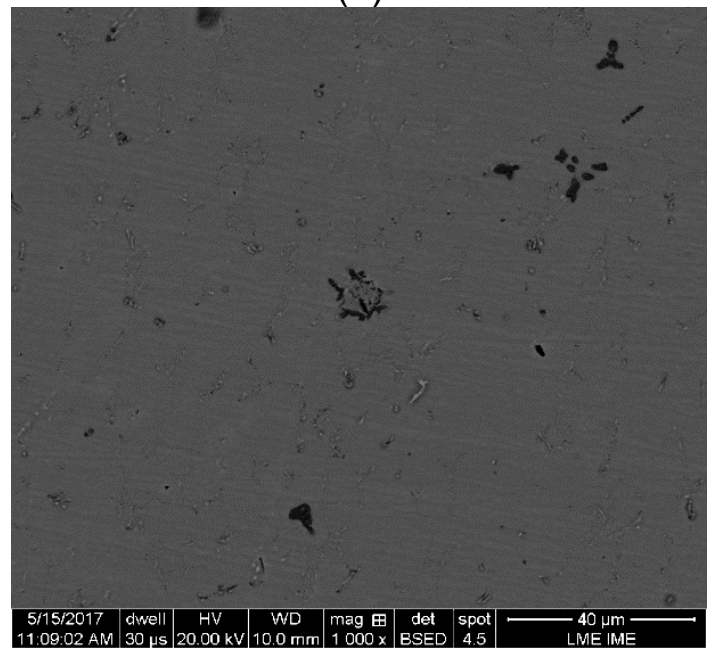

(d)

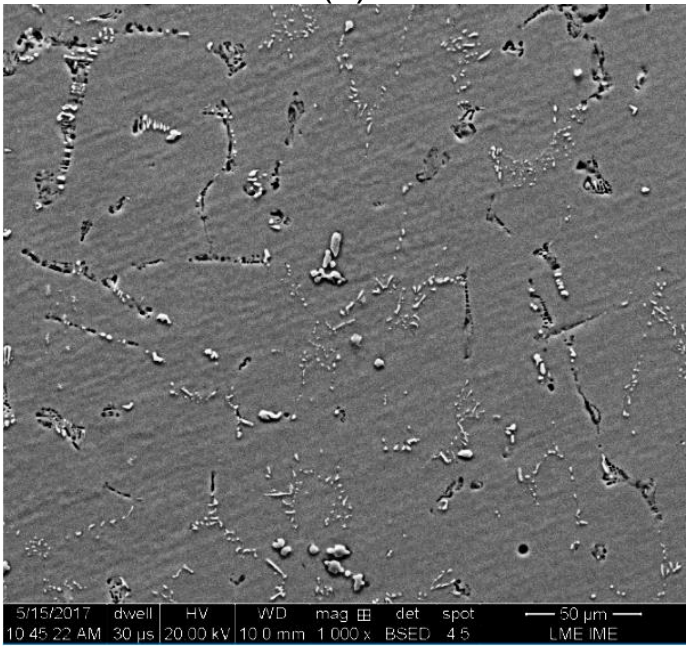

(f)

(e)

Figura 3. Imagens de MEV das amostras: (a) bruta de fusão (AM1); (c) refundida VAR ITA (AM3); e (e) refundida VAR UPT (AM6). Detectores de (a, c, e) SE e (b, d, f) BSE.

$\mathrm{Na}$ Figura 4 e Tabela 2 são apresentados os resultados de EDS vinculados as observações no MEV das microestruturas das imagens em estudo, onde selecionou como exemplo para cada uma das amostras uma região vinculada a característica 
típica das inclusões observadas nas mesmas a partir da sua observação geral da microestrutura (Figura 3).

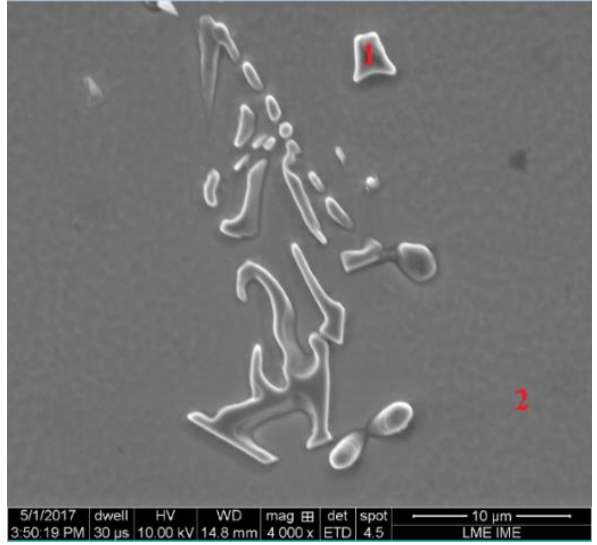

(a)

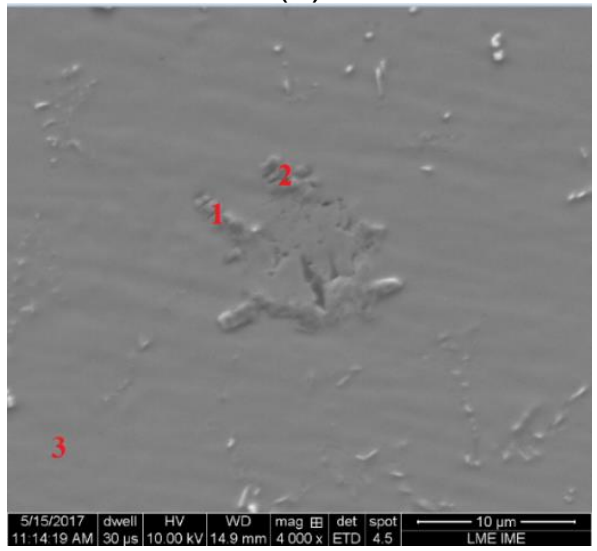

(c)

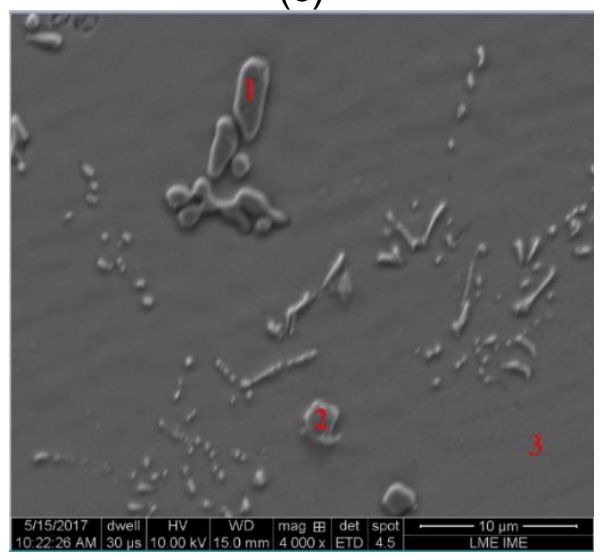

(e)

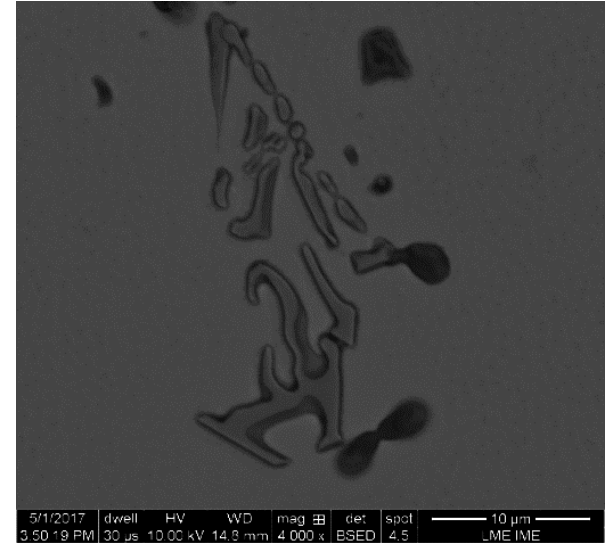

(b)

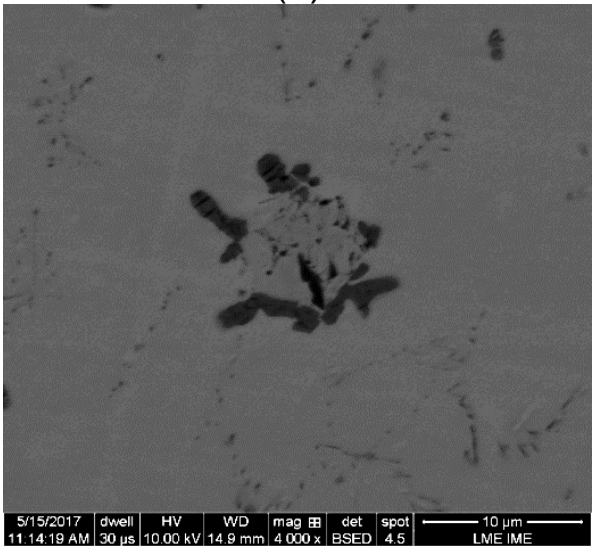

(d)

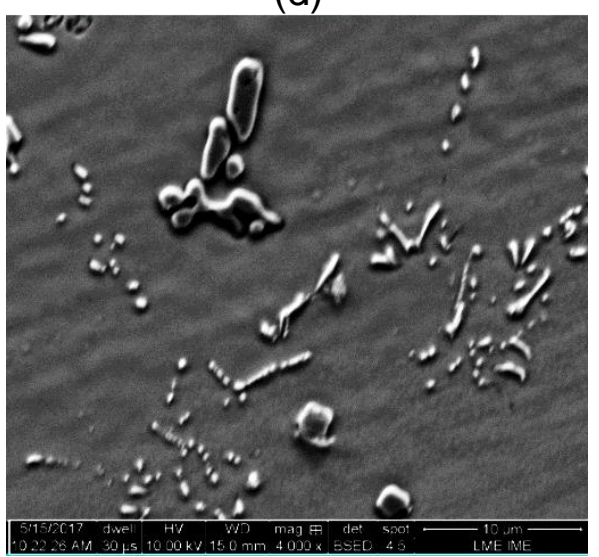

(f)

Figura 4. Imagens de MEV com maior aumento destacando as inclusões presentes nas amostras: (a) bruta de fusão (AM1); (c) refundida VAR ITA (AM3); e (e) refundida VAR UPT (AM6). Detectores de $(a, c, e)$ SE e (b, d, f) BSE. 
Tabela 2. Resumo da análise semi-quantitativa de EDS nos pontos destacada nas imagens apresentadas na Figura $4 \mathrm{a}, \mathrm{c}, \mathrm{e}$.

\begin{tabular}{|c|c|c|c|c|c|}
\hline \multirow{2}{*}{$\begin{array}{c}\text { Pontos } \\
\text { Analisados }\end{array}$} & \multirow{2}{*}{$\begin{array}{l}\text { Percentual em } \\
\text { peso e atômico }\end{array}$} & \multicolumn{4}{|c|}{ Elementos Detectados } \\
\hline & & $\mathrm{Ni}$ & $\mathrm{Ti}$ & 0 & C \\
\hline \multicolumn{6}{|c|}{ AM 1 - Figura 4a } \\
\hline \multirow[t]{2}{*}{1} & $\% p$ & 0,06 & 88,95 & 5,14 & 5,86 \\
\hline & \%at & 0,04 & 69,65 & 12,04 & 18,28 \\
\hline \multirow[t]{2}{*}{2} & $\% p$ & 43,36 & 53,48 & - & 3,16 \\
\hline & \%at & 34,87 & 52,72 & - & 12,41 \\
\hline \multicolumn{6}{|c|}{ AM 3 - Figura 4c } \\
\hline \multirow[t]{2}{*}{1} & $\% p$ & 2,81 & 83,60 & 4,63 & 8,96 \\
\hline & $\%$ at & 1,69 & 61,72 & 10,23 & 26,36 \\
\hline \multirow[t]{2}{*}{2} & $\% p$ & - & 90,79 & 2,51 & 6,70 \\
\hline & \%at & - & 72,63 & 6,02 & 21,35 \\
\hline \multirow[t]{2}{*}{3} & $\% p$ & 42,88 & 55,71 & - & 1,41 \\
\hline & \%at & 36,31 & 57,84 & - & 5,85 \\
\hline \multicolumn{6}{|c|}{ AM 6 - Figura $4 \mathrm{e}$} \\
\hline \multirow[t]{2}{*}{1} & $\% p$ & - & 86,64 & 6,66 & 8,70 \\
\hline & \%at & - & 60,79 & 14,31 & 24,90 \\
\hline \multirow[t]{2}{*}{2} & $\% p$ & 1,23 & 80,23 & 9,26 & 8,19 \\
\hline & \%at & 0,93 & 56,75 & 19,43 & 22,89 \\
\hline \multirow[t]{2}{*}{3} & $\% p$ & 43,94 & 54,74 & - & 1,32 \\
\hline & \%at & 37,38 & 57,10 & - & 5,52 \\
\hline
\end{tabular}

Pela análise de EDS/MEV (Figura 4), com uso de uma tensão de aceleração mais baixa $(10 \mathrm{kV})$, aumento superior (4000x) e WD elevado (aproximadamente $15 \mathrm{~mm}$ ) para prover um menor volume de interação amostra/feixe de elétrons, foi possível observar que as regiões/pontos mais claros evidenciados nas imagens apresentadas na Figura 3 indicam a presença de $\mathrm{Ni}, \mathrm{Ti}, \mathrm{C}$ e $\mathrm{O}$ (conforme extrato na Tabela 2) possivelmente associados à presença de carbonetos ( $\mathrm{TiC}$ ), óxidos $\left(\mathrm{Ti}_{4} \mathrm{Ni}_{2} \mathrm{O}\right.$ ) e/ou precipitados (p.ex. $\mathrm{Ni}_{4} \mathrm{Ti}_{3}$, identificado anteriormente na análise de DRX - Figura 2). Além da região mais escura de fundo, constituída de uma matriz metálica de NiTi associada possivelmente à fase B2. Conforme mencionado na literatura, amostra AM1 (oriunda de um processo VIM) com uma atmosfera não tão bem controlada, que $\mathrm{p}$ causando a incorporação de oxigênio durante a elaboração da liga no estado líquido [5]. Além disso, a carga metálica fundida entra em contato com o cadinho de grafite ocasionando a interdifusão e promovendo a formação de TiC devido a afinidade que o carbono tem de se combinar com titânio [7,15]. As amostras AM3 e AM6 (processo VAR) que foram produzidas a partir da AM1, com uma atmosfera melhor controlada, em termos de nível de vácuo prévio e purga posterior de gás argônio, mantêm o mesmo nível de contaminação originada da matéria prima (amostra AM1). Parte desse carbono é dissolvido e o restante permanece incorporado ao material devido seu ponto de fusão ser bem mais elevado. Por fim, a formação de precipitados na matriz da liga de NiTi, ocorre de acordo com a concentração que são difundidos o $\mathrm{Ni}$ e o Ti pelo metal líquido durante o processo de solidificação [19], em função da taxa de resfriamento imposta e o valor de material. Podendo tais fases serem confirmadas pela análise de DRX mostrada anteriormente (Figura 2).

De forma a complementar o estudo dos resultados apresentados estão sendo realizadas análises de mapeamento composicional via EDS/MEV e microtextural via difração de elétrons retroespalhados (EBSD/MEV), e associadas ao mapeamento das propriedades mecânicas em função da microestrutura com auxílio de um 
ultramicrodurômetro instrumentado com indentador Vickers com uso de baixas cargas.

\section{CONCLUSÃO}

Com base nos resultados analisados e sua respectiva discussão, com ênfase na liga em estudo, pode-se concluir que:

- A elaboração da liga via processo VIM em cadinho de grafite resultou na típica contaminação da mesma com $\mathrm{C}$ e $\mathrm{O}$, os quais foram identificados na forma de $\mathrm{TiC}$ e $\mathrm{Ti}_{4} \mathrm{Ni}_{2} \mathrm{O}$ conforme resultados de DRX associados a análise microestrutural de MEV juntamente com a análise semi-quantitativa de EDS;

- Aparentemente a refusão da liga via processo VAR com eletrodo não consumível (em atmosfera de argônio e com cadinho de cobre) não resultou no aumento do $\mathrm{O}$ incorporado a liga.

- A solidificação da liga em cadinhos de cobre promoveu um refinamento microestrutural tanto no que diz respeito a região de matriz metálica circundadas pela as inclusões como a redução no tamanho das inclusões.

\section{Agradecimentos}

Os autores agradecem ao professor J. Otubo por doar os materiais de partida, que foi elaborado no Instituto de Tecnologia Aeronáutica (São José dos Campos, SP Brasil) e uso do forno VAR. Como também ao professor Corneliu Craciunescu pela refusão no forno VAR da Departamento de Engenharia Mecânica (DEM) da Universidade Politécnica de Timisoara (UPT), através dos recursos do Projeto MIDAS fomentado pelo o programa Europeu de Ações Marie Curie.

F.S.S agradece a bolsa de mestrado subsidiada pela $\mathrm{CNPq}$ vinculada ao PGCM/SE4-IME. A.S.P. agracede a bolsa de produtividade à pesquisa (PQ-2) do CNPq (Processo 307798/2015-1). P.F.R e F.M.B.F reconhecem o financiamento da CENIMAT / i3N.

\section{REFERÊNCIAS}

1 MA, JL e WU, KH. Effects of Tantalum addition on transformation behaviour of $\left(\mathrm{Ni}_{51} \mathrm{Ti}_{49}\right.$ )$_{1-x} \mathrm{Ta}_{x}$ and $\mathrm{Ni}_{50} \mathrm{Ti}_{50-y} \mathrm{Ta}_{y}$ Shape Memory Alloys, Materials Science and Technology. 2000; volume (16): pp. 716-719.

2 MENG, XL, CAI, W, WANG, LM, ZHENG, YF, ZHAO, LC e ZHOU, LM. Microstructure of Stress-Induced Martensite in a Ti-Ni-Hf High. Temperature Shape Memory Alloy, Scripta Materialia. 2001; volume (45): pp.1177-1182.

$3 \mathrm{NOH}, \mathrm{JP}$. Phase transformation behaviours and shape memory characteristics of Ti(45$\mathrm{x}) \mathrm{Ni}-5 \mathrm{Cu}-\mathrm{xMo}(\mathrm{x}=0 \cdot 3-1 \cdot 0)$ alloys, Materials Science and Technology. 2001; volume (17): pp. 1544-1550.

4 TSAI, JCH e JEAN, RD. Effects of hot working on the martensitic transformation of NiTi alloy, Scripta Metallurgica et Materialia. 1994; volume (30); pp. 1027-1030.

5 SCHETKY, L e WU, MH. Issues in the further Development of Nitinol Properties and Processing for medical Device Applications, Memry Corporation, 2005; [acesso em18 out. 2010].Disponível em: http//memry.com/technology/pdfs/ASM03_NitinolDevelop.pdf

6 FRENZEL, J , ZHANG, Z , NEUKING, K e EGGELER, G. High quality vacuum induction melting of small quantities of NiTi shape memory alloys in graphite crucibles, Journal of Alloys and Compounds. 2004; volume (385): pp. 214-221. 
7 ZHANG, Z, FRENZEL, J, NEUKING, K e EGGELER, G. Vacuum induction melting of ternary $\operatorname{NiTiX}(X=\mathrm{Cu}, \mathrm{De}, \mathrm{Hf}, \mathrm{Zr})$ shape memory alloys using graphite crucibles, Materials Transactions A,. 2006; volume (47): pp. 661-665.

8 DAUTOVICH, DP e PURDY, GR. Phase Transformations in TiNi, Canadian Metallurgical Quarterly.1965; volume (4): pp. 129-134.

9 WU, MH. Fabrication of Nitinol materials and components, Proceedings of conference SMST -SMM 2001. 2001; pp. 285-291.

10 OLIVEIRA, G. C. M. Caracterização Microestrutural e Mecânica da Liga NiTi com EMF Produzida em Forno de Indução a Vácuo. 2010. Dissertação (Mestrado em Engenharia Aeronáutica e Mecânica - Área de Física e Química dos Materiais Aeroespaciais) Instituto Tecnológico de Aeronáutica, São José dos Campos, 2010.

11 RIBEIRO, Shimeni Baptista. Recuperação e recristalização de liga NiTi deformada por compressão e tratada termicamente no intervalo entre 150 e $500^{\circ} \mathrm{C}$, Tese de Doutorado, UFF/PPGEM, 2015.

12 OTSUKA, K e WAYMAN, CM. Mechanism of Shape Memory Effect and Superelasticity. In: Otsuka, K., Wayman, C. M. (eds.) Shape Memory Materials.Cambridge University Press, 1998; p. 01-26 e 27-48.

13 WANG, LM, LIU LH, YANG, H, WANG, LY e XIU, G.Q. Mater Sci Forum. 2002; 394$395 ; 297$.

14 JIANG, H., CAO, SH., KE., CH.; MA, X. e ZHANG, X. Fine-Grained Bulk NiTi shape Memory Alloy Fabricated by Rapid Process and Its Mechanical Properties and Damping Performance, Journal of Materials Science e Technology. 2013; volume (29): pp.855862.

$15 \mathrm{KOCICH}$, R., SZURMAN, I. e KURSA, M. The Methods of Preparation of Ti-Ni-X Alloys and Their Forming. 2013, Capítulo 2.

16 TALONEN J., ASPEGREN P. e HA"NNINEN H. Comparison of different methods for measuring strain induced a9-martensite content in austenitic steels. Materials Science and Technology,2004; volume (20): pp.1506.

17 ROZENAK P. e ELIEZER D.: MATER. SCI. ENG., 1984; 67, L1-L4.

18 FRENZEL J., ZHANG Z., SOMSEN CH., NEIKING K. e EGGELER G. Influence of carbon on martensitic phase transformations in NiTi shape memory alloys. Acta Materialia, 2007; volume (55): pp.1331-1341.

19 YANG, Z., TERRY,W. e SCHYVERS D. Scripta Mater, 52. 2005; pp.1129-1134. 